\title{
Hip Arthroscopy Has Acceptable Return to Sport Outcomes for the Elite Athlete
}

\author{
Iswadi Damasena ${ }^{1}$, Richard Jamieson ${ }^{2}$, Michael Pritchard ${ }^{2}$ \\ ${ }^{1}$ Department of Orthopaedic Surgery, Royal Perth Hospital, Perth, Australia; ${ }^{2}$ Department of Orthopaedic Surgery, Royal Hobart \\ Hospital, Hobart, Australia. \\ Email: i.damasena@hotmail.com
}

Received December $14^{\text {th }}, 2011$; revised January $25^{\text {th }}, 2012$; accepted February $12^{\text {th }}, 2012$

\begin{abstract}
Intra-articular disorders of the hip in the elite athlete are common and potentially career threatening. Hip arthroscopy has been shown to be a safe and successful method of treating these conditions. This study examines the effectiveness of hip arthroscopy in facilitating an early return to professional level sport. We prospectively followed 65 professional athletes (mostly Australian Rules Football players) before and after hip arthroscopy using the Modified Harris Hip Score (MHS) and the Non-Arthritic Hip (NAH) Score. We followed them to the 1 year mark and recorded the time required for them to return to sport. Follow-up was achieved in $100 \%$ of patients. Intraoperative findings included femoroacetabular impingement, labral pathology and ligamentum teres injuries. Both MHS and NAH Scores showed highly statistically significant improvements up to the 1 year mark. All but 3 athletes returned to professional level sport. The mean return to sport time was 11.9 weeks. The use of arthroscopy to manage common intra-articular hip disorders in elite athletes is safe, effective and facilitates an early return to sport.
\end{abstract}

Keywords: Arthroscopic Surgery; Hip Joint; Athletic Injuries

\section{Introduction}

Modern sport medicine aims to provide treatment that is less invasive in order to facilitate earlier return to competition. Demand for quick recovery and return to competition is high. Intra-articular disorders of the hip in the elite athlete are not uncommon and potentially career threatening.

Elite athletes subject their hips to significant peak-axial and torsional stress forces based on the nature of their activities [1]. This places them at greater risk of intraarticular hip disorders such as acetabularlabral tears, ligamentumteres injuries, and traumatic injury to the articular surface, amongst others [1-3].

Often, the level at which elite athletes perform exacerbate hip injuries that would otherwise be ignored in the general population. Such athletes commonly endure disabling symptoms, perform at sub-optimal levels, and undergo a multitude of tests before a source of injury is found. It is now known that there are numerous intraarticular sources of disabling hip pain potentially amenable to arthroscopic intervention [2,3].

The purpose of this study is to identify intra-articular hip disorders common to the elite athlete population and to demonstrate the effectiveness of hip arthroscopy to facilitate their return to competition at premorbid, or higher level of function within an acceptable timeframe. To our knowledge this is the largest study of its kind in elite athletes.

\section{Methods}

Sixty-five elite athletes (83 hips) were prospectively followed before and after hip arthroscopy. Elite athletes in this study were defined as those who were professional (i.e. paid) and were performing at either national or international level. Patients were selected based on their level of sport, their hip pain preventing them from competing, and their symptoms failing conservative therapy. All were diagnosed with hip pain potentially amenable to arthroscopic intervention based on a physical exam and radiographic data.

Follow up data was collected using the Modified Harris Hip score (MHS) and the Non-arthritic Hip (NAH) score. This included and assessment based on pain, symptoms, level of function, and physical activities. Scores were recorded 2 weeks prior to surgery and then at select intervals post-operatively at 2, 6, 12,26, and 52 weeks.

All procedures were performed by an experienced surgeon using the lateral decubitus position with the hip distracted by the McCarthy hip distractor attached to a regular operating table. The standard approach used a 
mid-lateral portal and an anterolateral portal, with a posterolateralportal used occasionally when required. All patients stayed overnight and were discharged the following day. All patients received one dose of $20 \mathrm{mg}$ subcutaneous enoxaparin for DVT prophylaxis, and one dose of antibiotics on induction. Post operatively patients were allowed to weight bear as tolerated and underwent a 12 week physiotherapy regime according to a standard protocol. They were followed up routinely by the surgeon at the 2 week mark, and at the 6, 12, 26, and 52 mark by mail. Return to sport was defined as the athlete resuming high level training for participation in their specific sport.

\section{Statistics}

Paired t-tests were utilized for comparison of means between pre- and post-operative MHS and NAH scores. Column graphs were used to depict mean trends in these scores. A p value of less than 0.05 (two-tailed) was considered statistically significant. All statistical analyses were performed on SPSS version 17.0 for Windows (SPSS Inc., Chicago, IL, USA).

\section{Results}

There were 83 hip arthroscopies (39 left-sided and 44 right-sided), including 18 bilateral procedures, performed on 65 professional and elite athletes (61 males and 4 females). The average age at time of surgery was 22.8 years, with a range of 16 to 39 years.

Follow-up has been achieved in $100 \%$ of patients, with a mean current follow-up period of 23 weeks. $42 \%$ of patients have currently been followed up to the 26 week mark and $22 \%$ have currently been followed to the 52 week mark (Figure 1).

Table 1 illustrates the types of sport played. The majority of patients (70.8\%) were Australian Rules Football players. From the remaining $29.2 \%$ cricket was the next most common followed by basketball, hockey, triathlon and long-distance running. There was one athlete from each of the sports of tennis, soccer, waterpolo, martial

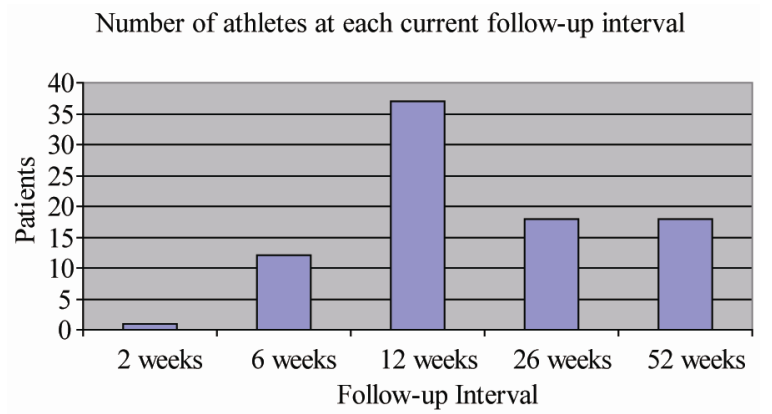

Figure 1. The number of patients currently at each followup period.
Table 1. Sporting category and the corresponding number of patients per category.

\begin{tabular}{lc}
\hline \multicolumn{1}{c}{ Sport } & Number \\
\hline Australian Rules Football & 46 \\
Cricket & 4 \\
Basketball & 2 \\
Triathlon & 2 \\
Hockey & 2 \\
Marathon running & 2 \\
Tennis & 1 \\
Soccer & 1 \\
Waterpolo & 1 \\
Martial arts & 1 \\
Circus Performing & 1 \\
Car Racing & 1 \\
Australian Football League umpire & 1 \\
\hline
\end{tabular}

arts, circus performing, car racing and umpiring.

The intra-operative findings and interventions are listed in Table 2. A significant number of patients had labralpathology. A radial tear was present in $39.7 \%$ and labral degeneration occurred in a further $16.8 \%$. A labral repair involved only the edge of the acetabulum and were superficial (i.e. not down to bone). Grade II lesions involved $<30 \%$ of the anterior width of the acetabulum, grade III lesions involved between $30 \%-50 \%$ of the acetabulum and grade IV lesions were $>50 \%$ of the acetabulum or had evidence of osteoarthritis. The vast majority $(62.7 \%)$ of patients had grade I lesions, $19.3 \%$ had grade II, $7.2 \%$ had grade III, and only 1 patient $(1.2 \%)$ had a grade IV lesion. In this particular case there was evidence of significant osteoarthritis.

A large proportion of patients $(41.0 \%)$ had a partial ligamentumteres tear with subsequent debridement performed.

A cam lesion was identified in $85.5 \%$. All of these patients underwent femoral neck ostectomy. A pincer lesion was identified in 10 patients $(12.0 \%) .25 .3 \%$ of patients underwent an acetabularostectomy for either a pincer lesion or those who had a significant labral tear.

Microfracture was performed in $22.9 \%$ of patients for focal chondral lesions. Three patients had adductor injections and 1 patient had bilateral tensor fascia latae injections.

Table 3 compares the mean scores at 2 weeks preoperatively with the post operative scores at 6, 12, 26 and 52 weeks. The 2 week postoperative scores were not included because there was no statistically significant change as it was too early in the postoperative recovery phase. The MHS showed a highly statistically significant improvement in mean scores at all postoperative intervals. 
Table 2. The intra-operative diagnoses and the interventions performed. The numbers represent the number of patients in each category.

\begin{tabular}{|c|c|c|c|}
\hline \multirow{2}{*}{$\begin{array}{l}\text { Intraoperative findings } \\
\text { Finding }\end{array}$} & \multicolumn{3}{|c|}{ Intraoperative intervention } \\
\hline & Number & Intervention & Number \\
\hline Mild synovitis & 7 & Femoral neck ostectomy & 71 \\
\hline Moderate synovitis & 2 & Acebular (rim) ostectomy & 21 \\
\hline Severe synovitis & 0 & Gluteus medius decompression/repair & 0 \\
\hline Labral pathology: & & Bursectomy & 0 \\
\hline Radial tear & 33 & Excision osacetabuli & 2 \\
\hline Degeneration & 14 & Loose chondromatosis & 0 \\
\hline Chondromalacia: & & Capsular release/capsulotomy & 12 \\
\hline Acetabular & 5 & Capsular repair & 4 \\
\hline Femoral head & 1 & Labral takedown & 26 \\
\hline Chondrocalcinosis & 0 & Labral repair & 26 \\
\hline Calcification & 0 & Labral debridement & 13 \\
\hline Osteochondritisdessicans & 0 & Autologous chrondrocyte implantation & 0 \\
\hline Rim lesions*: & & Microfracture & 19 \\
\hline Grade I & 52 & Psoas tenotomy & 0 \\
\hline Grade II & 16 & Ligamentumteres debridement & 34 \\
\hline Grade III & 6 & Tensor fascia lata injection & 2 \\
\hline Grade IV & 1 & Chondroplasty & 1 \\
\hline Osteoarthritis & 1 & Adductors injection & 3 \\
\hline Cam lesion & 71 & & \\
\hline Pincer lesion & 10 & & \\
\hline Avascular necrosis & 0 & & \\
\hline Femoral head cysts & 2 & & \\
\hline Ligament teres injury & 34 & & \\
\hline
\end{tabular}

"Defined as: Grade I lesion is superficial and involves edge of acetabulum only, grade II $<30 \%$ of anterior acetabulum width, grade III $30 \%$ - $50 \%$ of acetabulum, grade IV $>50 \%$ of acetabulum or osteoarthritis.

There was a mean increase in scores at 6 weeks of 9.1 (p $=0.001)$ and this was maintained throughout the follow-up period to a mean increase in score of 8.9 at 52 weeks $(p=0.036)$.

The total NAH score was subdivided into its subscores of pain, symptom, function and activities. The comparison of mean scores for pain, symptom and function, as well as the total score were all highly statistically significant throughout the entire follow-up period. There was no statistically significant difference in the activities scores at 6 weeks however this can be explained by the fact that most athletes had not yet returned to elite sport. There was, however, a statistically significant increase at the 12 week mark of 6 points $(p \leq 0.001)$, and this increase remained significant through to the final follow-up mark at 52 weeks.

Figures 2 and $\mathbf{3}$ demonstrate the mean MHS and the mean combined NAH score at preoperative and postoperative intervals respectively. Both showed the greatest improvement in the first 6 weeks followed by a plateau
Mean Modified Harris Hip Score at Pre- and Post-op Intervals

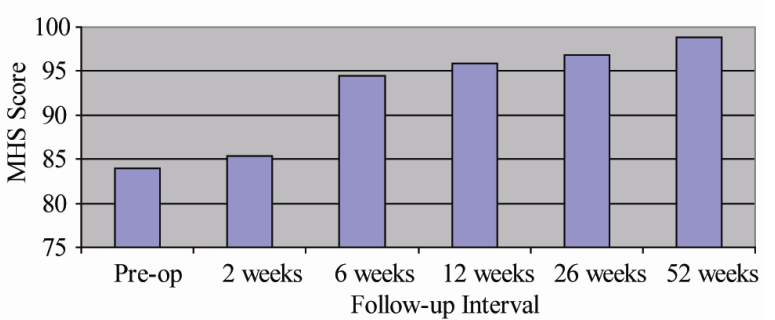

Figure 2. Mean Modified Harris Hip Scores at 2 weeks prehip arthroscopy, and at 2, 6, 12, 26, and 52 weeks post hip arthroscopy.

after about 12 weeks with a more gradual improvement to 52 weeks. Scores were significantly lower at 2 weeks reflecting a lighter workload in the immediate postoperative phase. As athletes became more active scores improved significantly thereafter.

Return to sport was achieved in $95.4 \%$ of athletes. Only 3 athletes did not return to sport and the reasons for 
Table 3. Shows the comparison of means at 2 weeks pre-hip arthroscopy and at intervals of 6 weeks, 12 weeks, 26 weeks, and 52 weeks post-hip arthroscopy for the Modified Harris Hip Score (MHS) and the Non-Arthritic Hip (NAH) Score. The NAH Scores are subdivided into pain, symptom, function, activities and total.

\begin{tabular}{|c|c|c|c|}
\hline \multicolumn{4}{|l|}{ MHS Hip Scores } \\
\hline Time intervals & Pre-op Mean (SD) & Post-op Mean (SD) & p value ${ }^{*}$ \\
\hline 2 Weeks Pre-op vs 6 Weeks Post-op & $84.2( \pm 14.9)$ & $93.3( \pm 7.7)$ & 0.001 \\
\hline 2 Weeks Pre-op vs 12 Weeks Post-op & $84.9( \pm 14.0)$ & $95.7( \pm 6.4)$ & $<0.001$ \\
\hline 2 Weeks Pre-op vs 26 Weeks Post-op & $84.4( \pm 15.0)$ & $96.7( \pm 7.7)$ & 0.008 \\
\hline 2 Weeks Pre-op vs 52 Weeks Post-op & $90.3( \pm 13.8)$ & $99.2( \pm 1.6)$ & 0.036 \\
\hline \multicolumn{4}{|l|}{ NAH PAIN Hip Scores } \\
\hline Time intervals & Pre-op Mean (SD) & Post-op Mean (SD) & p value ${ }^{*}$ \\
\hline 2 Weeks Pre-op vs 6 Weeks Post-op & $20.9( \pm 4.3)$ & $23.4( \pm 2.3)$ & 0.001 \\
\hline 2 Weeks Pre-op vs 12 Weeks Post-op & $20.2( \pm 4.4)$ & $24.1( \pm 1.8)$ & $<0.001$ \\
\hline 2 Weeks Pre-op vs 26 Weeks Post-op & $20.6( \pm 3.5)$ & $24.3(2.0)$ & $<0.001$ \\
\hline \multicolumn{4}{|l|}{ NAH SYMPTOM Hip Scores } \\
\hline Time intervals & Pre-op Mean (SD) & Post-op Mean (SD) & p value ${ }^{*}$ \\
\hline 2 Weeks Pre-op vs 6 Weeks Post-op & $13.8( \pm 3.8)$ & $17.7( \pm 2.2)$ & $<0.001$ \\
\hline 2 Weeks Pre-op vs 12 Weeks Post-op & $13.2( \pm 3.7)$ & $17.7( \pm 2.6)$ & $<0.001$ \\
\hline 2 Weeks Pre-op vs 26 Weeks Post-op & $14.0( \pm 3.1)$ & $18.0( \pm 2.5)$ & $<0.001$ \\
\hline 2 Weeks Pre-op vs 52 Weeks Post-op & $14.8( \pm 3.5)$ & $18.4( \pm 1.9)$ & 0.005 \\
\hline \multicolumn{4}{|l|}{ NAH FUNCTION Hip Scores } \\
\hline Time intervals & Pre-op Mean (SD) & Post-op Mean (SD) & p value ${ }^{*}$ \\
\hline 2 Weeks Pre-op vs 6 Weeks Post-op & $22.0( \pm 3.8)$ & $23.6( \pm 2.4)$ & 0.006 \\
\hline 2 Weeks Pre-op vs 12 Weeks Post-op & $21.6( \pm 3.7)$ & $23.8( \pm 2.8)$ & 0.004 \\
\hline \multicolumn{4}{|l|}{ NAH ACTIVITIES Hip Scores } \\
\hline Time intervals & Pre-op Mean (SD) & Post-op Mean (SD) & p value ${ }^{*}$ \\
\hline 2 Weeks Pre-op vs 6 Weeks Post-op & $21.2( \pm 5.5)$ & $21.6( \pm 5.9)$ & 0.737 \\
\hline 2 Weeks Pre-op vs 12 Weeks Post-op & $20.3( \pm 5.5)$ & $26.3( \pm 4.3)$ & $<0.001$ \\
\hline 2 Weeks Pre-op vs 26 Weeks Post-op & $21.1( \pm 5.0)$ & $28.3( \pm 3.1)$ & $<0.001$ \\
\hline 2 Weeks Pre-op vs 52 Weeks Post-op & $22.2( \pm 3.7)$ & $28.9( \pm 2.1)$ & $<0.001$ \\
\hline \multicolumn{4}{|l|}{ NAH TOTAL Hip Scores } \\
\hline Time intervals & Pre-op Mean (SD) & Post-op Mean (SD) & p value ${ }^{*}$ \\
\hline 2 Weeks Pre-op vs 6 Weeks Post-op & $78.2( \pm 14.4)$ & $86.7( \pm 9.8)$ & $<0.001$ \\
\hline 2 Weeks Pre-op vs 12 Weeks Post-op & $76.3( \pm 14.5)$ & $91.2( \pm 9.8)$ & $<0.001$ \\
\hline 2 Weeks Pre-op vs 26 Weeks Post-op & $78.4( \pm 11.2)$ & $94.8( \pm 7.7)$ & $<0.001$ \\
\hline 2 Weeks Pre-op vs 52 Weeks Post-op & $81.2( \pm 12.9)$ & $96.1( \pm 6.5)$ & 0.004 \\
\hline
\end{tabular}

*Determined by paired 2-tailed t-test comparison of means.

this are described in the discussion section. The average time required for the athletes to return to sport was 12.1 weeks. $77.0 \%$ returned to sport at or before the planned 12 week mark. The remaining $23.0 \%$ took between 13 and 26 weeks to return to sport, with the average time being 15.6 weeks. Only 3 patients from this group took longer than 16 weeks to return to elite level.

The earliest return to sport was seen in a racing car driver at the 6 week mark. 4 Australian Rules Football (AFL) players returned within 8 weeks and 1 marathon runner returned to high level training at the 9 week mark. Of the two majority sport types, the average return to 
Mean Non-Arthirtic Total hip scores at pre and post op intervals

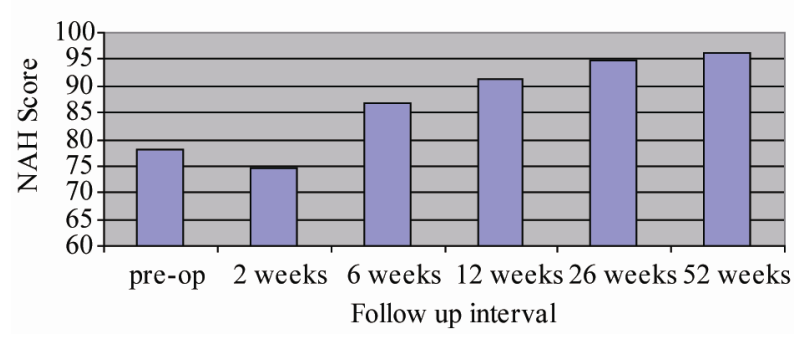

Figure 3. Mean Non-Arthritic Total Hip Scores at 2 weeks pre-hip arthroscopy, and at 2, 6, 12, 26, and 52 weeks post hip arthroscopy.

sport was 11.9 weeks for the AFL players and 12.5 weeks for the elite cricket players.

\section{Discussion}

The use of hip arthroscopy to treat intra-articular disorders of the hip in elite athletes is an evolving field that has become increasingly more popular $[1,3]$. The safety and efficacy of this procedure performed on athletes is now well recognised [1-5]. A recent publication by Byrd et al. has also showed good long-term results in the non-arthritic hip up to 10 years [6].

Arthroscopy can be used to treat a number of conditions commonly found in the athletic hip including labral tears, loose bodies, femoroacetabular impingement (FAI) and injuries of the ligamentumteres [7-9]. All of these conditions were present in our group of athletes. FAI was the most prevalent of these with greater than $80 \%$ of patients undergoing femoral ostectomy for a cam lesion. FAI is now a well-described condition and if left untreated has been shown to lead to osteoarthritis of the hip [10]. Among athletes, it is a major source of hip pain, reduced range of motion and decreased performance [2].

Labral pathology was present in over half of the patients. Traumatic labral tears in athletes are often thought to be caused by chronic repetitive activities, particularly hyperflexion and the lower limb stances associated with sporting activities $[1,2]$. Labral repair may therefore not only help to alleviate hip pain but also slow the progression of osteoarthritis in the joint [11].

A significant proportion of patients in our study (41.0\%) had ligamentumteres injuries. Tears of the ligamentumteres may be an under recognised source of chronic hip pain and has been suggested to be the third most common reason for hip pain in athletes [2,9].

A number of athletes had significant acetabularchondral lesions and microfracture was performed in this instance. Microfracture has been shown to be effective in reducing the size of the defect, particularly in patients with grade I-II rim lesions [12,13].
The aim of our study was to evaluate the efficacy of hip arthroscopy in returning elite athletes to professionallevel sport. To our knowledge, very few studies have demonstrated these findings and all have lower patient numbers [1,14-19]. Bharam studied 28 professional athletes but focused only on athletes with known labral tears and showed the earliest return was seen in golfers with a mean time of 6 weeks, followed by hockey players and skaters. Baseball and soccer players had a mean return time of 12 weeks. This study also concluded that one of the reasons for delayed returned to sport was the extended period of protected weight bearing required after the management of bony abnormalitiesassociated with labral tears including FAI and developmental conditions [14].

A study by Philippon et al. showed promising results with $93 \%$ of athletes having returned to professional sport [15]. This study did not, however, quantify the length of time required and also only included athletes with previously diagnosed FAI. McCarthy et al. evaluated the outcome of hip arthroscopy in 10 elite athletes, 7 of which were professional hockey players. All athletes successfully returned to sport although the timeframe was not published [1]. A more recent publication by Philippon et al. examined the outcomes of arthroscopic labral repair and treatment of FAI in 28 professional hockey players. They showed a mean MHS improvement of 25 and an average return to sport time of 3.4 months [16].

Nho et al. reported on a mixed group of athletes undergoing treatment for FAI and found 78\% had returned to sport at the 12 month mark [17]. Perhaps the mostcomparitive study was by Singh et al. who demonstrated a high satisfaction rate amongst 24 Australian rules football (AFL) players who underwent arthroscopy for hip pathology [18]. They found all had returned to training by the 3 month mark but did not specify the mean duration of return to sport.

A study by Larson et al. found that treatment of athletic pubalgia in addition to intra-articular disorders led to improved postoperative outcomes and unrestricted return to sportin patients who were symptomatic for both [19]. Patients either underwent concurrent treatment of both or staged procedures if indicated. Interestingly those who underwent hip arthroscopy alone fared much better than those who underwent surgery for athletic pubalgia, $69 \%$ of them re-presenting and undergoing arthroscopic surgery after failing to return to sport.

Our study focuses on early return to sport and includes all arthroscopic intra-operative findings including FAI, labral pathology and ligamentumteres injuries. The majority of our patients were AFL players. This is a high intensity contact sport that subjects players' hips to significant peak-axial and torsional stress forces. We have 
shown that hip arthroscopy in these athletes is successful, with a mean return to sport time of 11.9 weeks. In some cases this figure may slightly over-estimate the actual return to sport time for a single hip because a significant proportion $(27.7 \%)$ of these athletes underwent sequential bilateral hip arthroscopies.

Only three athletes in this cohort did not return to sport. One had a coexisting pubic symphysis infection with ongoing pain, another had concurrent knee and shoulder injuries preventing him from returning to sport, and the third athlete was found to have significant osteoarthritis at the time of primary hip arthroscopy and as a result made the decision to retire from sport. Four athletes were excluded due to inadequate follow-up times. All four had undergone hip arthroscopy within 2 weeks of data collection and hence had not yet had a 2 week postoperative follow-up appointment. Three patients had superficial wound infections that resolved after a short course of oral antibiotics. There were no other surgical complications.

Our findings in this report are subject to at least three limitations. Firstly our subjects were all elite athletes, similar conclusions may not apply to lower level athletes or novice sportmen and women. Secondly, pathologies varied amongst the athletes and hence their intra-operative intervention. Therefore a direct comparison to a group with a specific type of pathology cannot be made. Finnally, our study focuses on time taken to retun to competitive sport, it remains to be seen if these athletes maintain their unrestricted activity long term. Future studies should focus on specific intra-articular disorders andexamine the long term benefit of their treatment with respect to return to sport, career prolongation and the effect it has on modifying the natural progression to degenerative joint disease.

\section{Conclusion}

The use of hip arthroscopy to treat elite athletes with intra-articular disorders of the hip is becoming increasingly more popular. This study quantitatively assessed athletes in a prospective manner and demonstrates a statistically significant increase in both the MHS and the NAH score. Almost all athletes had returned to professional level sport by the 3 month mark. To our knowledge, we have the largest number of patients in the literature and have showed hip arthroscopy to be a safe and effective treatment option that can provide a timely return to sport for professional athletes.

\section{REFERENCES}

[1] J. McCarthy, W. Barsoum, L. Puri, J. A. Lee, S. Murphy and P. Cooke, "The Role of Hip Arthroscopy in the Elite Athlete," Clinical Orthopaedics and Related Research, Vol. 406, No. 1, 2003, pp. 71-74.

\section{doi:10.1097/00003086-200301000-00012}

[2] M. K. Shindle, J. E. Voos, B. E. Heyworth, D. N. Mintz, L. E. Moya, R. L. Buly and B. T. Kelly, "Hip Arthroscopy in the Athletic Patient: Current Techniques and Spectrum of Disease," The Journal of Bone and Joint Surgery American, Vol. 89, No. S3, 2007, pp. 29-43. doi:10.2106/JBJS.G.00603

[3] J. W. Byrd and K. S. Jones, "Hip Arthroscopy in Athletes," Clinics in Sport Medicine, Vol. 20, No. 4, 2001, pp. 749-761. doi:10.1016/S0278-5919(05)70282-9

[4] B. T. Kelly and R. L. Buly, "Hip Arthroscopy Update," Hospital for Special Surgery Journal, Vol. 1, No. 1, 2005, pp. 40-48.

[5] M. J. Philippon, "Arthroscopy of the Hip in the Management of the Athlete. In Operative Arthroscopy, 3rd Edition, Williams \& Wilkins, Philadephia, 2009, pp. 879883.

[6] J. W. Byrd and K. S. J. ones, "Hip Arthroscopy in Athletes: 10-Year Follow-Up," American Journal of Sports Medicine, Vol. 37, No. 11, 2005, pp. 2140-2143. doi: $10.1177 / 0363546509337705$

[7] S. Bharam, "Labral Tears, Extra-Articular Injuries, and Hip Arthroscopy in the Athlete," Clinics in Sports Medicine, Vol. 25, No. 2, 2006, pp. 279-292. doi:10.1016/j.csm.2006.01.003

[8] M. J. Philippon and M. Schenker, "Arthroscopy for the Treatment of Femoroacetabular Impingement in the Athlete," Clinics in Sports Medicine, Vol. 25, No. 2, 2006, pp. 299-308. doi:10.1016/i.csm.2005.12.006

[9] N. V. Bardakos and R. N. Villar, "The Ligamentumteres of the Adult Hip," Journal of Bone and Joint Surgery British Volume, Vol. 91, No. 1, 2009, pp. 8-15. doi:10.1302/0301-620X.91B1.21421

[10] R. Ganz, J. Parvizi, M. Beck, M. Leunig, H. Notzli and K. Siebenrock, "Femoroacetabular Impingement: A Cause for Osteoarthritis of the Hip," Clinics on Orthopedics and Related Research, Vol. 417, 2003, pp. 112-120.

[11] J. McCarthy, P. Noble, M. Schuck, J. Wright and J. Lee, "The Otto E. Aufranc Award: The Role of Labral Lesions to Development of Early Degenerative Hip Disease," Clinics in Orthopedics and Related Research, Vol. 393, 2001, pp. 25-37. doi:10.1097/00003086-200112000-00004

[12] M. J. Philippon, M. Schenker, K. Briggs and R. Maxwell. "Can Microfracture Produce Repair Tissue in Acetabularchondral Defects?" Arthroscopy, Vol. 24, No. 1, 2008, pp. 46-50. doi:10.1016/j.arthro.2007.07.027

[13] K. Crawford, M. J. Philippon, J. Sekiya, W. Rodkey and J. Steadman, "Microfracture of the Hip in Athletes," Clinics in Sports Medicine, Vol. 25, No. 2, 2006, pp. 327-335. doi:10.1016/i.csm.2005.12.004

[14] S. Bharam, V. Kandemir, P. Dravitch, F. Fu and M. J. Philippon, "Return to Competition in Professional Athletes with Traumatic Labral Tears of the Hip," Proceedings from the 28th Annual Meeting of American Orthopaedic Society of Sports Medicine, Orlando, 10 February 2002.

[15] M. J. Philippon, M. Schenker, K. Briggs and D. Kup- 
persmith, "Femoroacetabular Impingement in 45 Professional Athletes: Associated Pathologies and Return to Sport Following Arthroscopic Decompression," Knee Surgery, Sports Traumatology, Arthroscopy, Vol. 15, No. 7, 2007, pp. 908-914.

[16] M. J. Philippon, D. Weiss, D. Kuppersmith, K. Briggs and C. Hay, "Arthroscopic Labral Repair and Treatment of the Femoroacetabular Impingement in Professional Hockey Players," American Journal of Sports Medicine, Vol. 38, No. 1, 2010, pp. 99-104. doi:10.1177/0363546509346393

[17] S. J. Nho, E. M. Magennis, C. K. Singh and B. T. Kelly, "Outcomes after the Arthroscopic Treatment of Femo- roacetabular Impingement in a Mixed Group of HighLevel Athletes," American Journal of Sports Medicine, Vol. 39, No. S1, 2011, pp. 14S-9S.

[18] P. J. Singh and J. M. O'Donnell, "The Outcome of Hip Arthroscopy in Australian Rules Football League Players: A Review of 27 Hips," Arthroscopy, Vol. 26, No. 6, 2010, pp. 743-749. doi:10.1016/j.arthro.2009.10.010

[19] C. M. Larson, B. R. Pierce and M. R. Giveans, "Treatment of Athletes with Symptomatic Intra-Articular Hip Pathology and Athletic Pubalgia/Sports Hernia: A Case Series," Arthroscopy, Vol. 27, No. 6, 2011, pp. 768-775. doi:10.1016/j.arthro.2011.01.018 